\title{
DESEMPENHO INDIVIDUAL DE COMPÓSITOS REFORÇADOS COM FIBRAS DE CURAUÁ SOB IMPACTO BALÍSTICO COM MUNIÇÃO 7,62 MM*
}

Fábio de Oliveira Braga ${ }^{1}$ Sérgio Neves Monteiro²

\section{Resumo}

Atualmente, tecidos de fibras sintéticas como a aramida (Kevlar®) e o polietileno (Dyneema®) têm sido os preferidos para proteção balística contra munições leves, e para integrar sistemas de proteção contra calibres superiores, como o 7,62 mm. Porém, por apelos econômicos, ambientais e da disponibilidade limitada destes produtos, materiais alternativos têm sido testados. Neste cenário, destacam-se os compósitos reforçados com fibras naturais, que têm demonstrado bom desempenho balístico. Assim, o objetivo deste trabalho é avaliar o desempenho individual de compósitos reforçados com fibras naturais de curauá, quando submetidos ao impacto balístico com munição $7,62 \mathrm{~mm}$. Foram executados ensaios balísticos, medindo-se a energia do projétil absorvida pelos materais, e os resultados tratados estatisticamente por Anova e teste de Tukey. Mostrou-se que estes compósitos têm potencial para substituir as fibras sintéticas em sistemas de blindagem para proteção pessoal, com a vantagem de serem materiais leves, de baixo custo, nacionais, de ampla disponibilidade e ambientalmente corretos.

Palavras-chave: Blindagem balística; Fibras naturais; Fibras de curauá; Compósitos.

\section{INDIVIDUAL PERFORMANCE OF COMPOSITES REINFORCED WITH CURAUA FIBERS UNDER BALLISTIC IMPACT WITH 7.62 MM AMMUNITION}

\begin{abstract}
Currently, synthetic fiber woven fabrics as aramid (Kevlar®) and polyethylene (Dyneema®) have been the favorite materials for ballistic protection against light ammunition, and to integrate protection systems against superior caliber as 7,62 $\mathrm{mm}$. However, due to economic and environmental issues, and limited availability of these products, alternative materials have been tested. In this scenario, natural fiber reinforced composites, that have demonstrated good ballistic performance, stand out. Thus, the objective of this work is to evaluate the individual performance of natural curaua reinforced composites, when subjected to ballistic impact with $7.62 \mathrm{~mm}$ ammunition. Ballistic tests were performed, measuring the absorbed energy of the projectile, and the results were statistically treated by Anova and Tukey's test. It was shown that these composites have the potential to substitute synthetic fibers in personal protection armor systems, with advantages of being light, low cost, local materials, of wide availability and environmentally friendly.
\end{abstract}

Keywords: Ballistic armor; Natural fibers; Curaua fibers; Composites.

1 Engenheiro Metalurgista, M.Sc., Doutorando em Ciência dos Materiais, Seção de Engenharia Mecânica e de Materiais, Instituto Militar de Engenharia, Rio de Janeiro, RJ, Brasil. fabio_obraga@yahoo.com.br.

2 Engenheiro Metalurgista, Ph.D, Professor Titular, Seção de Engenharia Mecânica e de Materiais, Instituto Militar de Engenharia, Rio de Janeiro, RJ, Brasil. 


\section{INTRODUÇÃO}

Á medida que a tecnologia de armas e munições progride, maiores se tornam os desafios para os engenheiros e pesquisadores da área de materiais para blindagens balísticas. Cada vez mais, materiais de alto desempenho são necessários, mas a busca pela melhor performance deve levar em conta também a mobilidade que os combatentes e os sistemas de defesa devem possuir. O design ótimo de um sistema de blindagem deve balancear estas características conflitantes ${ }^{1}$.

Atualmente, tecidos de fibras sintéticas como a aramida (Kevlar®) e o polietileno de ultra-alto peso molecular (Dyneema®) têm sido os preferidos para proteção balística contra munições leves, e para integrar sistemas de proteção contra calibres superiores, como o 7,62 $\mathrm{mm}^{1-3}$. No entanto, em virtude de apelos econômicos, ambientais e da disponibilidade limitada destes produtos, diversos materiais alternativos têm sido testados para estas aplicações.

Neste cenário, destacam-se os compósitos reforçados com fibras naturais, que têm demonstrado bom desempenho balístico, ao integrarem os chamados sistemas de blindagem multicamada, que utilizam diferentes materiais em sua estrutura ${ }^{3-4}$.

Entre as fibras naturais, destacam-se as fibras de curauá, em virtude da sua alta resistência e rigidez, e do baixo peso e alta tenacidade dos seus compósitos. As fibras de curauá são obtidas das folhas do Ananas erectifolius, planta nativa da região amazônica ${ }^{3}$.

Existem diversos métodos de avaliação do desempenho balístico de um material para blindagem, como a medida da velocidade limite do projétil na blindagem $\left(\mathrm{V}_{50}\right)^{4}$, a medida da deformação de plástica um material de referência ${ }^{2-3}$, bem como medidas de energia absorvida pela blindagem ${ }^{2,3,5}$. Este último permite uma avaliação individual rápida de materiais de naturezas distintas, fornecendo algumas informações importantes acerca do seu comportamento balístico. Desta forma, o objetivo deste trabalho é avaliar o desempenho individual de compósitos reforçados com fibras de curauá, quando submetidos ao impacto balístico com munição 7,62 $\mathrm{mm}$.

\section{MATERIAIS E MÉTODOS}

A Tabela 1 mostra os materiais a serem testados neste trabalho e suas respectivas designações. Além dos compósitos reforçados com fibras de curauá, serão testados alguns materiais de uso comum para blindagens, como o alumínio e a aramida.

Tabela 1. Tipo e designação dos materiais examinados no presente trabalho

\begin{tabular}{cc}
\hline Tipo & Designação \\
\hline Poliéster & P-0\%C \\
\hline $\begin{array}{c}\text { Poliéster reforçado com } 10 \% \text { vol. de } \\
\text { fibras de curauá }\end{array}$ & P-10\%C \\
\hline $\begin{array}{c}\text { Poliéster reforçado com } 20 \% \text { vol. de } \\
\text { fibras de curauá }\end{array}$ & P-20\%C \\
\hline $\begin{array}{c}\text { Poliéster reforçado com } 30 \% \text { vol. de } \\
\text { fibras de curauá }\end{array}$ & P-30\%C \\
\hline $\begin{array}{c}\text { Laminado com 16 camadas de } \\
\text { aramida }\end{array}$ & Aramida \\
\hline Liga de alumínio 5052 H34 & Alumínio \\
\hline
\end{tabular}


As fibras de curauá foram fornecidas pela empresa Pematec Triangel. A resina poliéster, por sua vez, foi fornecida pela empresa Resinpoxy, sendo comercializada como resina poliéster Cristal. Esta foi misturada ao endurecedor na proporção de $1 \%$ em peso, e também às fibras previamente secas em estufa (24h), em uma matriz de aço, mantendo-se sob pressão até a cura (temperatura ambiente, 24h). Os compósitos fabricados possuem a forma de placas retangulares de dimensões $119 \times 150 \times 10.0 \mathrm{~mm}$, nas proporções de 0,10,20 e 30\% em volume de fibras alinhadas, designados por $\mathrm{P}-0 \% \mathrm{C}, \mathrm{P}-10 \% \mathrm{C}, \mathrm{P}-20 \% \mathrm{C}$ e $\mathrm{P}-30 \% \mathrm{C}$, respectivamente (Tabela 1). Foram produzidas duas placas de cada composição, e cada uma foi dividida em quatro placas menores, resultando em oito placas para o ensaio balístico.

A placa liga de alumínio 5052 H34 foi fornecida pela empresa Metalak Metais, nas dimensões $120 \times 150 \times 4,76 \mathrm{~mm}$. Algumas de suas características são mostradas na Tabela 2.

Tabela 2. Características da liga de alumínio utilizada

\begin{tabular}{|c|c|c|c|c|c|}
\hline Propriedade mecânica & Méc & & \multicolumn{3}{|c|}{ Desvio padrão } \\
\hline Resistência à tração (MPa) & 233 & & \multicolumn{3}{|c|}{4,1} \\
\hline Deformação total (\%) & 18 , & & \multicolumn{3}{|c|}{1,9} \\
\hline Dureza (HRB) & 29 , & & \multicolumn{3}{|c|}{ - } \\
\hline Composição química $^{\mathrm{c}}$ & Al & Mg & Fe & Si & $\mathrm{Cr}$ \\
\hline Teor dos elementos (\%) & Balanço & 2,25 & 0,32 & 0,20 & 0,16 \\
\hline
\end{tabular}

O tecido de aramida utilizado neste trabalho, de trama $S 745$ e gramatura $460 \mathrm{~g} / \mathrm{m}^{2}$, foi fornecido pela empresa LFJ Blindagens, Comércio e Serviços S.A. (Conquext), sob a forma de painéis de 8 camadas impregnadas com borracha cloroprênica (modelo MENEOKV08). Em cada corpo de prova balístico, foram unidos dois painéis MENEOKV08 com adesivo à base de poliuretano, a fim de que a espessura fosse próxia a $10 \mathrm{~mm}$.

Os materiais foram submetidos ao impacto balístico. O dispositivo de disparo, disponível no Centro de Avaliações do Exército (CAEx), consistiu de um provete com mira à laser (Figura 1a) e um radar doppler para medição da velocidade do projétil (Figura 1b). Foi utilizada munição 7,62 mm M1 (Figura 1c), com massa de $9.7 \mathrm{~g}$, comercialmente fornecida ao Exército Brasileiro. O provete estava localizado a $15 \mathrm{~m}$ do alvo (material a ser testado, Figura 1d), conforme previsto na norma ABNT $15000^{6}$. O disparo foi realizado na horizontal $\left(\theta=0^{\circ}\right)$. A energia cinética absorvida pela blindagem ( $E_{a b s}$ ), foi utilizada para comparação entre os materiais, sendo calculada pela equação 1 .

$$
E_{a b s}=\frac{m\left(v_{i}^{2}-v_{r}^{2}\right)}{2}
$$

Onde: $\mathrm{m}=$ massa do projétil; $\mathrm{vi}_{\mathrm{i}}=$ velocidade de impacto do projétil na blindagem; $v_{r}=$ velocidade residual do projétil após o impacto.

Sete disparos foram realizados para cada material, e foram descartados o maior e o menor valor de energia absorvida obtido. 


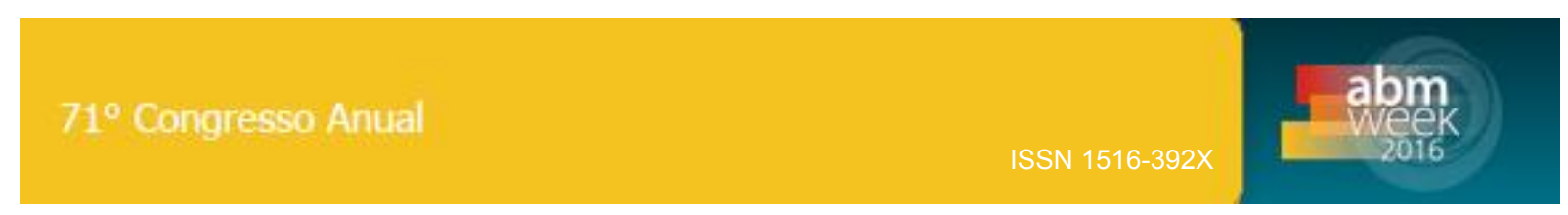

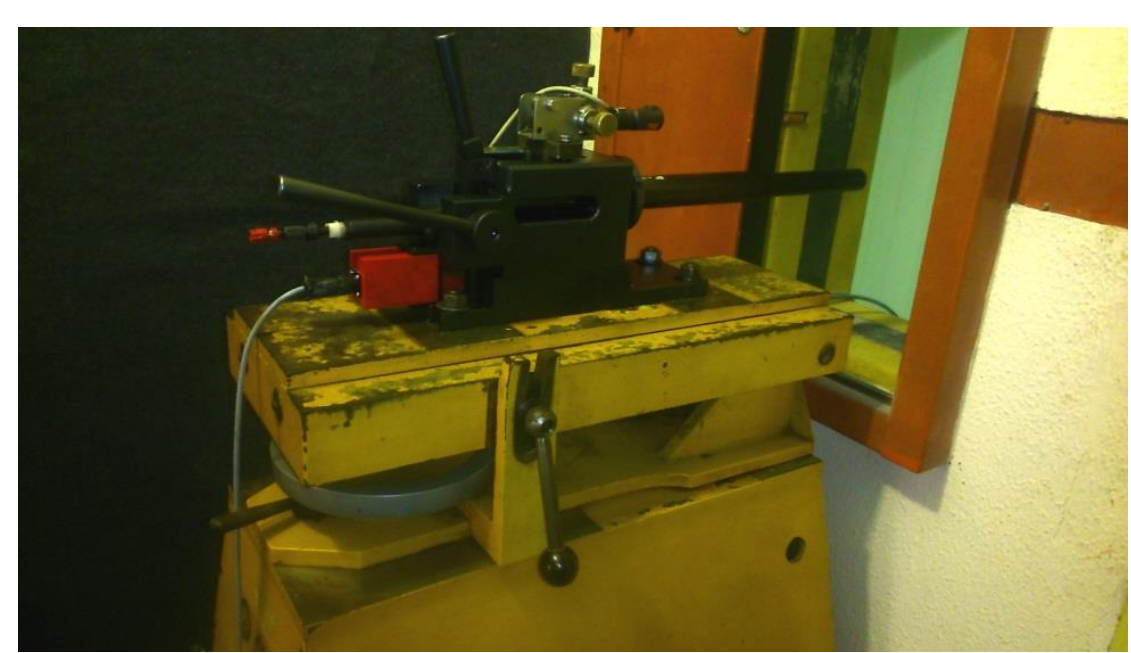

(a)

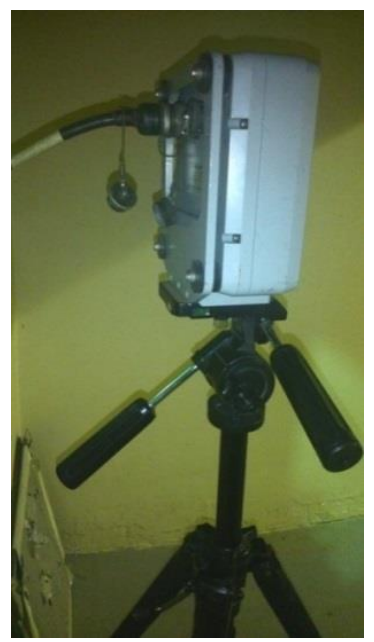

(b)

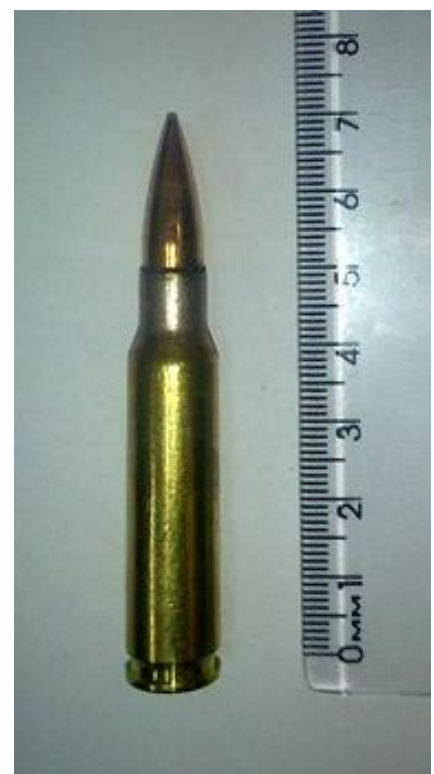

(c)

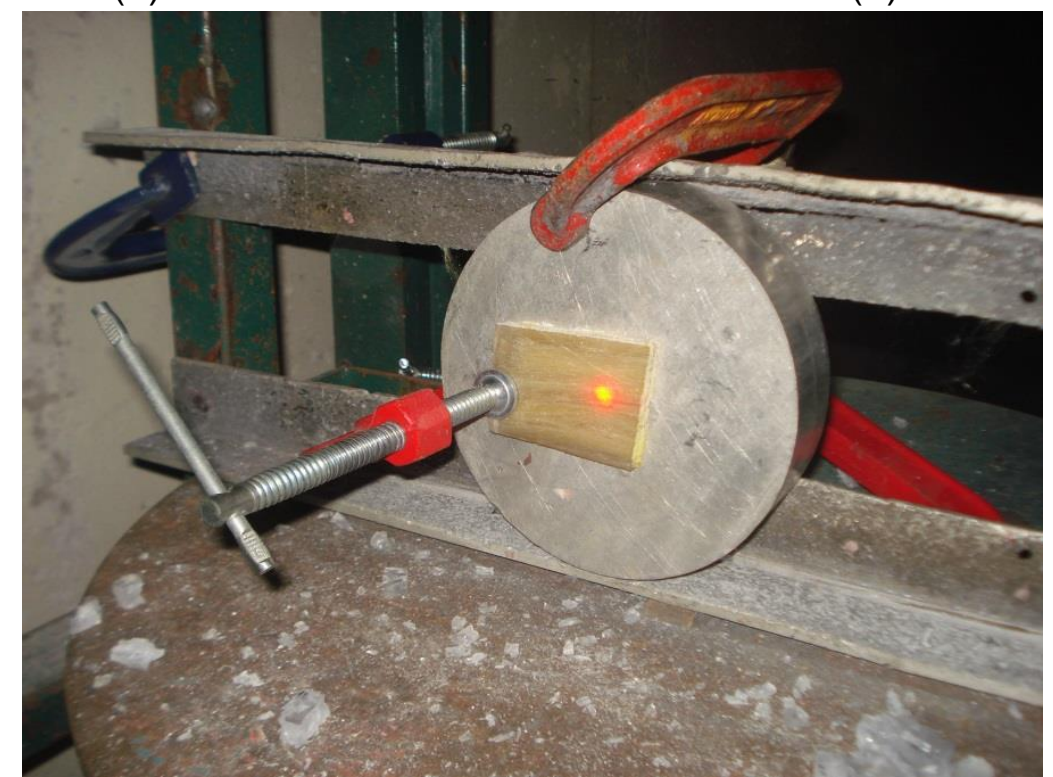

(d)

Figura 1. Arranjo experimental do ensaio balístico: (a) provete com mira à laser; (b) radar doppler; (c) munição 7,62 mm M1; (d) corpo de prova montado à frente de bloco de alumínio com orifício central.

Os valores de energia absorvida foram tratados estatisticamente utilizando-se a análise de variância (Anova), seguida pela comparação das médias pelo teste de Tukey, calculando-se a diferença mínima de significância (d.m.s.) pela equação $2^{7}$.

$$
\text { d.m.s }=q \sqrt{\frac{Q M R}{r}}
$$

Onde: $q$ = constante tabelada para $5 \%$ de significâcia; QMR = quadrado médio do resíduo da análise de variância; $r=5=$ número de repetições de cada tratamento.

\section{RESULTADOS E DISCUSSÃO}

A Tabela 3 mostra os valores de energia absorvida pelos diferentes materiais submetidos ao impacto do projétil $7,62 \mathrm{~mm}$. A princípio, verifica-se que o tipo de 
material (tratamento) influencia na energia absorvida, mas a confirmação deverá vir da análise de variância.

Tabela 3. Energia absorvida pelo material mediante o impacto balístico

\begin{tabular}{ccccccc}
\hline & \multicolumn{7}{c}{ Material } \\
\cline { 2 - 7 } & $\mathbf{P - 0 \% C}$ & $\mathbf{P - 1 0 \% C}$ & $\mathbf{P - 2 0 \% C}$ & $\mathbf{P - 3 0 \% C}$ & Aramida & Alumínio \\
\hline & 230 & 150 & 147 & 194 & 201 & 227 \\
\cline { 2 - 7 } $\begin{array}{c}\text { Energia } \\
\text { absorvida } \\
(\mathrm{J})\end{array}$ & 260 & 156 & 156 & 197 & 221 & 234 \\
\cline { 2 - 7 } & 267 & 190 & 163 & 200 & 226 & 240 \\
\cline { 2 - 7 } & 268 & 224 & 164 & 218 & 229 & 249 \\
\hline $\begin{array}{c}\text { Energia } \\
\text { média (J) }\end{array}$ & $\mathbf{2 5 9}$ & $\mathbf{2 0 2}$ & $\mathbf{1 6 5}$ & $\mathbf{2 0 8}$ & $\mathbf{2 2 3}$ & $\mathbf{2 4 3}$ \\
\hline
\end{tabular}

A Tabela 4 mostra a análise de variância dos dados, sendo apresentados os graus de liberdade (GL), as somas de quadrados (SQ), os quadrados médios (QM), o $F$ de Snedecor e o parâmetro $F$ crítico $\left(F_{c}\right)$ tabelado.

Tabela 4. Análise de variância dos dados

\begin{tabular}{lccccc}
\hline Causas de variação & GL & SQ & QM & F & $\mathbf{F}_{\mathbf{c}}$ \\
\cline { 1 - 3 } Tratamentos & 5 & 26997 & 5399 & 6,98 & 2,62 \\
\cline { 1 - 4 } Resíduo & 24 & 18565 & 773 & & \\
\cline { 1 - 4 } Total & 29 & 45562 & & & \\
\hline
\end{tabular}

Comparando-se o valor de $\mathrm{F}$ com $\mathrm{F}_{\mathrm{c}}$, rejeita-se a hipótese de que as médias dos tratamentos são iguais, com $95 \%$ de confiança, uma vez que $\mathrm{F}>\mathrm{Fc}$. Em outras palavras, o tipo de material utilizado influencia na energia absorvida no impacto do projétil, sendo confirmada a impressão obtida anteriormente.

Tendo sido completada a análise de variância, é possível fazer a comparação das médias individuais pelo teste de Tukey. 0 valor de q para 6 tratamentos e 24 graus de liberdade para o resíduo é 4,37. Desta forma a d.m.s. foi calculada pela equação 2 como $54 \mathrm{~J}$. A Tabela 5 mostra a comparação entre as médias pelo teste de Tukey.

Tabela 5. Comparação entre as médias pelo teste de Tukey. Os valores das diferenças de energia são dados em Joules. Os valores maiores que d.m.s. são apresentados em verde e negrito, e, do contrário, apresentados em vermelho

\begin{tabular}{lcccccc}
\hline Material & $\mathbf{P - 0} \% \mathbf{C}$ & $\mathbf{P - 1 0} \% \mathbf{C}$ & $\mathbf{P - 2 0} \% \mathbf{C}$ & $\mathbf{P - 3 0} \% \mathbf{C}$ & Aramida & Alumínio \\
\hline $\mathbf{P - 0} \% \mathbf{C}$ & 0 & $\mathbf{5 7}$ & $\mathbf{9 3}$ & 51 & 35 & 15 \\
\hline $\mathbf{P - 1 0} \% \mathbf{C}$ & $\mathbf{5 7}$ & 0 & 36 & 6 & 21 & 41 \\
\hline $\mathbf{P - 2 0} \% \mathbf{C}$ & $\mathbf{9 3}$ & 36 & 0 & 42 & $\mathbf{5 8}$ & $\mathbf{7 8}$ \\
\hline $\mathbf{P - 3 0} \% \mathbf{C}$ & 51 & 6 & 42 & 0 & 16 & 36 \\
\hline Aramida & 35 & 21 & $\mathbf{5 8}$ & 16 & 0 & 20 \\
\hline Alumínio & 15 & 41 & $\mathbf{7 8}$ & 36 & 20 & 0 \\
\hline
\end{tabular}

Pelo exame das Tabela 3 e 5, observa-se que o material P-0\%C foi o de melhor resultado, provavelmente devido à sua característica frágil, que dissipa energia na criação de superfícies de fratura, como ocorre na fragmentação das cerâmicas ${ }^{2}$. A placa $\mathrm{P}-0 \% \mathrm{C}$ foi totalmente fragmentada, como pode ser observado na Figura 2a, que mostra alguns fragmentos recuperados. Apesar da maior absorção de energia, a total fragmentação pode ser um problema caso o cenário seja de múltiplos impactos. No outro extremo, os materiais $\mathrm{P}-10 \% \mathrm{C}$ e $\mathrm{P}-20 \% \mathrm{C}$ tiveram o pior desempenho balístico. $O$ teste de Tukey confirmou que estas duas composições pioram a 
absorção de energia do projétil em relação a $\mathrm{P}-0 \% \mathrm{C}$. Isto se deve, provavelmente, à pequena quantidade de fibras no compósito, que leva a uma perda da continuidade da matriz polimérica, sem que haja um reforço efetivo. Isto pode ser observado na Figura $2 b$, que mostra a delaminação, ou a separação das fibras da matriz nos corpos de prova $\mathrm{P}-10 \% \mathrm{C}$.

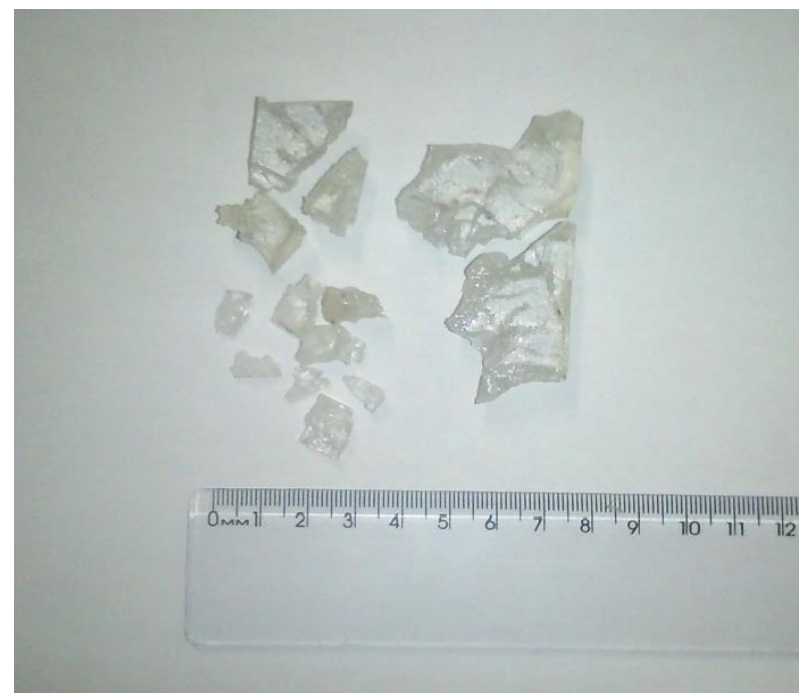

(a)

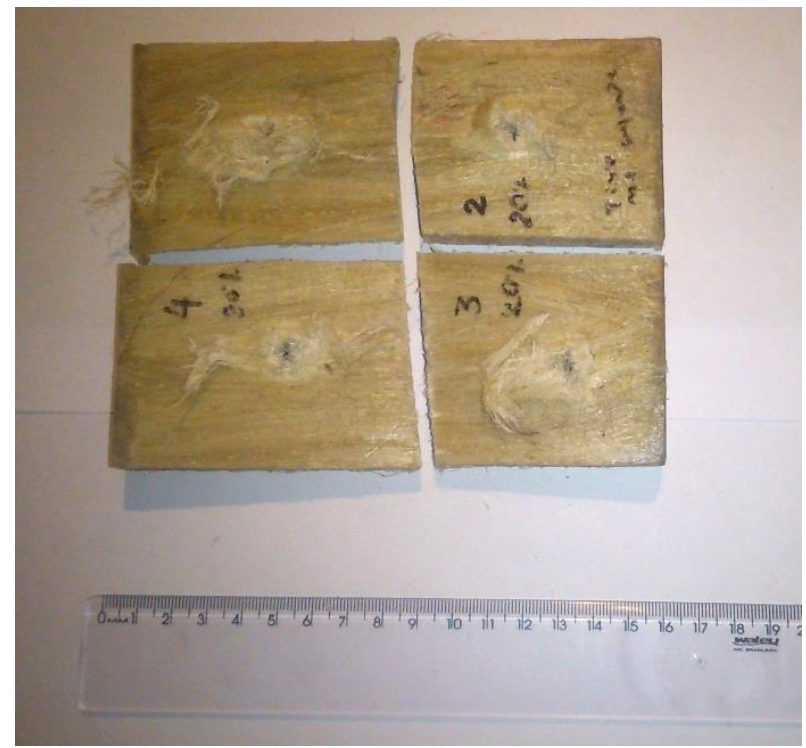

(c)

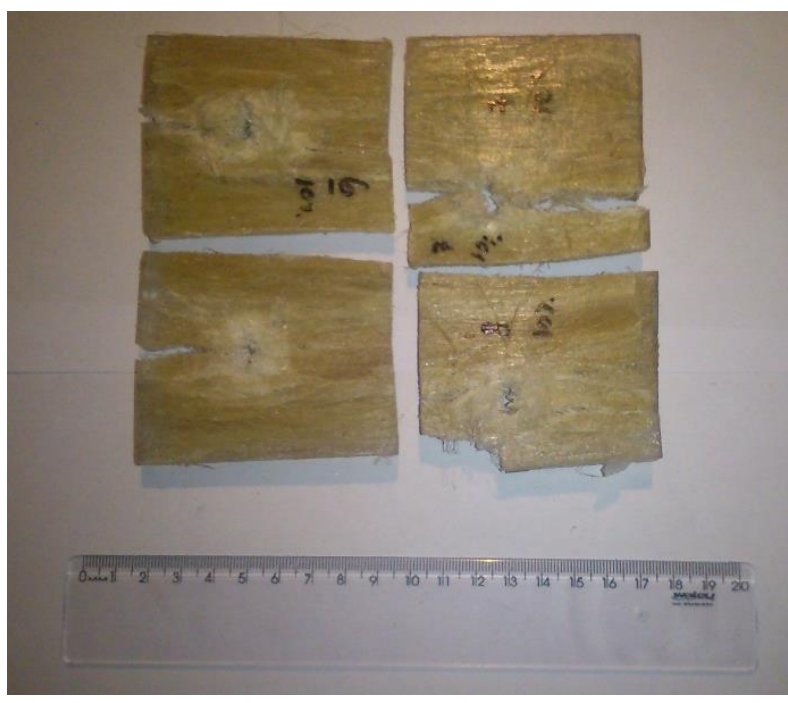

(b)

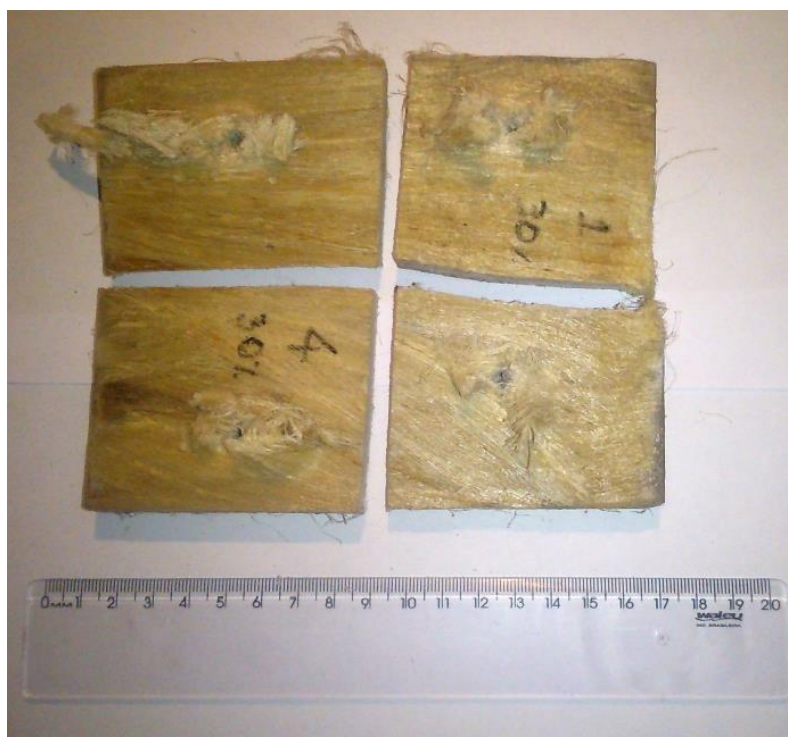

(d)

Figura 2. Corpos de prova submetidos ao impacto balístico: (a) P-0\%C; (b) P-10\%C; (c) P-20\%C; (d) $\mathrm{P}-30 \% \mathrm{C}$. 
O compósito $\mathrm{P}-30 \% \mathrm{C}$ foi o de melhor resultado, sendo sua média comparável às da aramida, alumínio e P-0\%C, como mostrou o teste de Tukey (Tabela 5). A Figura 2d mostra o aspecto do corpo-de-prova após o ensaio. Apesar de o compósito P-30\%C ter absorvido uma quantidade um pouco menor de energia nos ensaios que o $\mathrm{P}$ 0\% , o fato de manter sua integridade mediante ao impacto balístico é de grande importância para integrar sistemas de blindagens para munição $7,62 \mathrm{~mm}$ (multicamada), principalmente em uma eventual segunda camada, com a finalidade de absorver os fragmentos de um material cerâmico frontal2-3. Assim, considera-se que este material tem propriedades aceitáveis para integrar um sistema de blindagem, com a vantagem de ser leve, de baixo custo, nacional, de ampla disponibilidade e ambientalmente correto.

\section{CONCLUSÃO}

A partir dos resultados obtidos, pode-se concluir que os compósitos reforçados por fibras de curauá tem potencial para substituir as fibras sintéticas em sistemas de blindagem para proteção pessoal. A proporção de fibras que obteve melhor desempenho balístico foi $30 \%$ em volume, considerando que seus compósitos de matriz poliéster tiveram boa absorção de energia do projétil $7,62 \mathrm{~mm}$, em relação aos materiais utilizados na atualidade, e mantiveram sua integridade após o impacto, sendo adequados para múltiplos impactos. Assim, considera-se que este material tem propriedades aceitáveis para integrar um sistema de blindagem, com a vantagem de ser leve, de baixo custo, nacional, de ampla disponibilidade e ambientalmente correto.

\section{Agradecimentos}

Os autores deste trabalho agradecem ao CNPq pelo financiamento da pesquisa, através do processo 159753 de 2015-5, e ao CAEx pela execução dos ensaios balísticos.

\section{REFERÊNCIAS}

1 Wang L, Kanesalingan S, Nayak R, Padhye R. Recent trends in ballistic protection. Textiles and Light Industrial Science and Technology. 2014; 3:37-47.

2 Monteiro SN, Lima Jr. EP, Louro LHL, Silva LC, Drelich JW. Unlocking function of aramida fibers in multilayered ballistic armor. Metallurgical and Materials Transactions. A, Physical Metallurgy and Materials Science. 2014; 46:37-40.

3 Monteiro SN, Louro LHL, Trindade W, Elias CN, Ferreira CL, Lima ES, Weber RP, Suarez JCM, Figueiredo ABS, Pinheiro WA, Silva LC, Lima EP. Natural curaua fiberreinforced composites in multilayered ballistic armor. Metallurgical and Materials Transactions. A, Physical Metallurgy and Materials Science. 2015; 46:4567-4577. Wambua P, Vangrimde B, Lomov S, Verpoest I. The response of natural fibre composites to ballistic impact by fragmente simulating projectiles. Composite Structures. 2007; 77:232-240.

5 Morye SS, Hine PJ, Duckett RA, Carr DJ, Ward IM. Modelling of the energy absorption by polymer composites upon ballistic impact. Composites Science and Technology. $2000 ; 60: 2631-2642$.

6 ABNT NBR 15000. Blindagens para impactos balísticos - classificação e critérios de avaliação. 2005.

7 Vieira S. Análise de Variância (Anova). São Paulo: Ed. Atlas; 2006. 